\title{
Acesso aos serviços de saúde: comparação da visão de profissionais e usuários da atenção básica
}

\author{
Health service access: comparing professionals' and users' views of primary care \\ El acceso a los servicios de salud: comparando la visión de profesionales y usuarios de la \\ atención primaria
}

\author{
Maria José Sanches Marin'; Maria Yvette Aguiar Dutra MoracvickiI; Milton MarchiolilI
}

\begin{abstract}
RESUMO: No presente estudo descritivo propôs-se a comparar o acesso aos serviços de saúde na ótica de usuários e profissionais que atuam na estratégia saúde da família (ESF) e nas unidades básicas de saúde tradicionais (UBST) de um município do interior paulista. Foram realizadas 32 entrevistas e aplicados dois questionários com 17 assertivas; um deles respondido por 396 usuários e o outro, por 289 profissionais, em 2011 e 2012. Na análise quantitativa, tanto usuários como profissionais emitiram respostas que tendem à positividade, exceto quanto ao período de funcionamento da unidade e tempo de espera para consulta médica. Na análise qualitativa, as opiniões de profissionais e usuários se aproximaram na maioria dos aspectos e divergiram, principalmente, no que se refere à atenção básica como porta de entrada para o sistema e à necessidade de contrarreferência. Conclui-se pela necessidade de mudanças na concepção de atendimento à saúde.

Palavras-Chave: Acesso aos serviços de saúde; serviços de saúde; atenção primária à saúde; pessoal de saúde.
\end{abstract}

\begin{abstract}
This descriptive study compared the views of health professionals and users of the Family Health Strategy and traditional basic health units as regards health service access in a municipality in São Paulo State. Thirty-two interviews were conducted and two 17-response questionnaires applied, one answered by 396 users and the other by 289 professionals, in 2011 and 2012. In the quantitative analysis, both professionals and users tended to respond favorably, except as regards health facility opening hours and appointment waiting times. In the qualitative analysis, professionals' and users' opinions were close on most issues, diverging mainly as regards primary health care as the system gateway, and the need for counter-referral. It was concluded that changes are needed in the conception of health care.
\end{abstract}

Keywords: health service access; health services; primary health care; health personnel.

RESUMEN: Este estudio descriptivo objetivó comparar el acceso a los servicios de salud bajo la visión de usuarios y profesionales que trabajan en la estrategia salud de la familia (ESF) y en las unidades básicas de salud tradicionales (UBST) en un municipio del Estado de São Paulo-Brasil. Fueron realizadas 32 entrevistas y se aplicaron dos cuestionarios con 17 asertivas, uno de ellos respondido por 396 usuarios y el otro por 289 profesionales, en 2011 y 2012. En el análisis cuantitativo, tanto los profesionales cuanto los usuarios han emitido respuestas que tienden a la positividad, excepto cuanto al período de funcionamiento de la unidad y tiempo de espera para consulta médica. En el análisis cualitativo, las opiniones de profesionales y de usuarios fueron muy semejantes en la mayoría de los aspectos y discordaron principalmente cuanto a la atención básica como entrada en el sistema y la necesidad de contrareferencia. Se concluyó por la necesidad de cambios en la concepción del atendimiento a la salud.

Palavras Clave: Accesibilidad a los servicios de salud; servicios de salud; atención primaria a la salud; personal de salud.

\section{INTRODUÇÃO}

Na proposição do atual Sistema Único de Saúde (SUS), definiu-se um conceito ampliado de saúde. Nele se consideram os diferentes determinantes e condicionantes do processo de saúde/doença, estabelecendo que a atenção à saúde deve seguir os princípios da universalidade do acesso, integralidade da assistência, equidade, descentralização, hierarquização e participação social ${ }^{1}$, com ênfase na atenção primária.
Referindo-se aos preceitos constitucionais que garantem o direito de todos os cidadãos a uma assistência à saúde em conformidade com os princípios e as diretrizes do SUS, o acesso aos serviços de saúde representa um marco de referência abrangente para o estudo dos seus avanços e das dificuldades, podendo ser considerado o primeiro passo na busca do atendimento às necessidades de saúde da população.

'Doutora em Enfermagem e Docente do Curso de Enfermagem da Faculdade de Medicina de Marília. São Paulo, Brasil. E-mail: marnadia@terra.com.br IIMédica Dermatologista e Docente do Curso de Medicina da Faculdade de Medicina de Marília. São Paulo, Brasil. E-mail: somebody@terra.com.br IIIDoutor em Medicina. Docente da Disciplina de Educação em Ciências da Saúde da Faculdade de Medicina de Marília. São Paulo, Brasil. E-mail: miltonmachioli@unimedmarilia.com.br

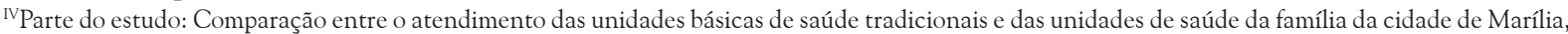
na ótica dos usuários e profissionais. 
Frente a tais preceitos e considerando a necessidade de verificação dos avanços e dos desafios impostos a esse acesso, o objetivo deste estudo ${ }^{I V}$ foi comparar aspectos do acesso aos serviços de saúde na ótica de usuários e de profissionais que atuam na estratégia saúde da família (ESF) e nas unidades básicas de saúde tradicionais (UBST).

\section{REVISÃO DE LITERATURA}

O acesso aos serviços de saúde tem sido alvo de diferentes interpretações. $\mathrm{O}$ conceito mais abrangente, no entanto, envolve disponibilidade, acessibilidade, aceitabilidade e capacidade financeira ${ }^{2}$.

No estudo da atenção primária, destacam-se, como importantes indicadores da qualidade, o acesso, a porta de entrada, o elenco de serviços e a coordenação ou a integração entre eles ${ }^{3}$. Compreende-se acesso como a localização da unidade de saúde próxima da população à qual atende, os horários e os dias em que está aberta para atender, o grau de tolerância para consultas não-agendadas e o quanto a população percebe essa conveniência.

A porta de entrada refere-se à utilização do serviço de atenção básica como o primeiro atendimento procurado, por meio do qual as pessoas buscam atenção à saúde, exceto nos casos de urgência. $\bigcirc$ elenco de serviços indica os arranjos que a unidade realiza para que o usuário receba a atenção de que necessita.

Quanto à coordenação ou integração de serviços, contemplam-se a facilidade em acessar os demais níveis de atenção e o acompanhamento do atendimento em outros serviços especializados. Tais aspectos relacionamse às possibilidades de os usuários conseguirem, no momento desejado, o atendimento a suas necessidades de saúde, compreendendo que o sistema se organiza de forma hierarquizada e que, embora a porta de entrada possa resolver grande parte dos problemas de saúde, ela não dispõe de tecnologias apropriadas para atender à integralidade do cuidado.

A questão do acesso compreende quatro dimensões de análise: a econômica refere-se à relação entre a oferta e a demanda pelos serviços de saúde; a técnica exprime o planejamento e a organização da rede de serviços para concretizar o atendimento pretendido pelo usuário; a política diz respeito à capacidade de desenvolvimento da consciência sanitária e da organização social; e a simbólica é relativa às representações sociais acerca da atenção e à organização do sistema de saúde para atender às necessidades dos usuários. Para se trabalhar o acesso aos serviços de saúde, deve-se englobar a maior quantidade e qualidade possível de variáveis relacionadas ${ }^{4}$.

Nessa perspectiva, o acesso aos serviços de saúde envolve elementos que se configuram como condição essencial à transformação da realidade e ao atendimento aos direitos de cidadania ${ }^{5}$, além de representar um grande avanço, pois historicamente o atendimento à saúde tem sido negligenciado pelas políticas públicas, especialmente para as pessoas mais carentes.

$\mathrm{O}$ acesso universal a todas as tecnologias de saúde, como um direito adquirido, precisa de acompanhamento sistemático com vistas à sua qualificação, embora o seu significado seja amplo e envolva a concepção e a demanda das pessoas e da comunidade aos serviços. Acredita-se que somente por meio da experimentação e da avaliação seja possível avançar na utilização dos cuidados em saúde, principalmente entre os menos favorecida ${ }^{2}$.

Nessa direção, estudo nacional evidenciou a presença de desigualdades no acesso a serviços saúde em distintas regiões, além de heterogeneidade interna, manifestada principalmente em relação à renda e à faixa etária ${ }^{6,7}$. Destacam-se também barreiras organizacionais nas unidades, refletidas pelo perfil dos profissionais que nelas atuam ${ }^{8}$.

\section{Metodologia}

A presente investigação compara o atendimento da ESF e das UBST na ótica dos profissionais e dos usuários em um município do interior paulista. Foram utilizadas as abordagens de pesquisa qualitativa e quantitativa na perspectiva da complementaridade. O município em tela está localizado na região centrooeste do Estado de São Paulo, com uma população aproximada de 220.000 habitantes. O sistema de atenção básica conta com 12 UBST e 30 unidades de saúde da família (USF). As USF do município atendem, aproximadamente, a 50\% da população da cidade. Para a realização do estudo selecionaram-se quatro UBST e 12 USF, por meio de sorteio, sendo uma UBST e três USFs de cada região da cidade (norte, sul, leste e oeste).

A coleta de dados, realizada no período de dezembro de 2011 a fevereiro de 2012, pautou-se nas condições de acesso, na porta de entrada, no elenco de serviços e na integração entre esses. Os dados qualitativos foram coletados por meio de entrevistas com perguntas semiestruturadas. A seleção dos entrevistados buscou contemplar, entre os usuários, pessoas adultas de diferentes faixas etárias, portadoras de agravos e pessoas sem agravos no estado de saúde; entre os profissionais, foram contempladas as diferentes áreas de formação. Assim, realizaram-se 32 entrevistas, sendo oito com usuários e oito com profissionais das UBST; oito com usuários e oito com profissionais da ESF. Todas as entrevistas foram gravadas e transcritas na íntegra.

A análise desses dados qualitativos foi fundamentada na hermenêutica-dialética, cujos parâmetros 
de interpretação são os seguintes: leitura compreensiva do contexto em que os dados são gerados e criação de estruturas de análise em que se buscam as ideias que estão por trás dos textos 9 . Trabalha-se com sentidos mais amplos, caminhando para um movimento de síntese por meio da construção de possíveis significados.

Os dados quantitativos foram coletados a partir de dois instrumentos, um deles destinado ao usuário e o outro aos profissionais. Cada um deles contou com 17 assertivas. Para obter a opinião dos participantes, foi utilizada a Escala de Likert, baseada no critério de ocorrência (1. Nunca; 2. Quase nunca; 3. Algumas vezes; 4. Muitas vezes; 5. Quase sempre; 6. Sempre), na qual usuários e profissionais de saúde especificam seu nível de concordância com a afirmação.

A amostra populacional para obtenção dos dados quantitativos foi obtida a partir do cálculo de amostra aleatória simples, que permite a representatividade da população, com a possibilidade de erro amostral de, mais ou menos, $5 \%{ }^{10}$. Assim, ao utilizar a fórmula

$$
n=\left(\frac{z_{\alpha / 2} \sqrt{p(1-p)}}{s}\right)^{2}
$$

em que $z_{\alpha / 2}=1,95$ (correspondente ao quantil 95\% da distribuição normal padrão), $\mathrm{p}=0,5$ e $\varepsilon=0,05$, obtém-se uma amostra de 400 usuários. Concordaram em participar deste estudo 396 clientes. Por meio do cálculo amostral dos profissionais de saúde, obtevese uma amostra de 150 da UBS e 164 da ESF, pois atuam nelas 240 e 280 profissionais, respectivamente. Aderiram ao estudo 289 profissionais.

A aplicação do instrumento foi realizada por pessoas devidamente treinadas pelos pesquisadores. $\mathrm{O}$ questionário destinado aos profissionais foi autoaplicado, após as devidas orientações para o preenchimento. Os usuários que tinham condições de ler e compreender o conteúdo responderam ao questionário sem a ajuda do responsável pela coleta e, para aqueles que não tinham tal condição, foi realizada a leitura e solicitado que emitissem respostas.

Os dados quantitativos foram digitados em planilhas do Excel 2000 for Windows, seguidos de análises estatísticas realizadas com o software Statistical Package for Social Sciences (SPSS) versão 11.0 for Windows. As análises inferenciais, empregadas com intuito de verificar a associação da avaliação entre os usuários da ESF e da UBST, bem como dos profissionais das duas modalidades, foram realizadas por meio do Teste Wilcoxon ${ }^{11}$. Em todas as conclusões obtidas pelas análises inferenciais foi utilizado o nível de significância igual a $5 \%(\mathrm{p} \leq 0,005)$.

A pesquisa contou com a autorização do Secretário Municipal de Saúde e com a aprovação do Comitê de Ética e Pesquisa que Envolve Seres Humanos, da Faculdade de Medicina de Marília, sob protocolo $n^{\circ} 682 / 08$. Todos os sujeitos que aceitaram participar do estudo assinaram o Termo de Consentimento Livre e Esclarecido (TCLE). Para garantir o anonimato dos participantes entrevistados, eles foram identificados pela letra U (Usuário) ou P (Profissional), numerados de acordo com a sequência das entrevistas, de 1 a 8, seguido do local da realização (UBST ou ESF).

\section{Resultados e Discussão}

Quanto às características sociodemográficas, 95,2\% dos profissionais que participaram do estudo são do sexo feminino; 32\%, agentes comunitários de saúde; 22,6\%, auxiliares ou técnicos de enfermagem; $9,3 \%$, enfermeiros; $5,3 \%$ e médicos, entre outras categorias. Quanto ao tempo de serviço, obteve-se, em média, 10,9 anos para os profissionais da UBST e 6,1 anos para os profissionais da ESF. Referindo-se aos usuários, a idade variou de 18 a 83 anos, com uma média de 44,2 anos, sendo 71,7\% do sexo feminino.

Observa-se que, na ótica dos usuários das duas modalidades de assistência, a ESF obteve média mais favorável na maioria das assertivas. Quanto aos itens dois e cinco, é importante salientar que as respostas alcançaram médias de pontuações mais baixas, conforme mostra a Tabela 1 .

Ao se compararem as respostas dos profissionais das ESFs e das UBSTs quanto à condição de acessibilidade aos serviços de saúde, constatou-se que apenas em três assertivas houve diferenças estatisticamente significativas, sendo que os profissionais das UBSTs tendem a uma avaliação mais positiva dos aspectos analisados, segundo a Tabela 2 .

$\mathrm{Na}$ análise qualitativa, ao se considerarem os fatores relacionados à acessibilidade aos serviços de saúde na ótica dos usuários e dos profissionais das USFs e das UBSTs, foi possível identificar três núcleos de sentido, conforme é analisado a seguir.

\section{Adequações e inadequações do acesso}

Na ótica dos participantes do estudo, a localização da unidade não representa problema para nenhuma das modalidades. Já, quanto à disponibilidade de horário para atendimento médico, os usuários apontam para a frequente falta de profissional e para a necessidade de chegarem muito cedo à unidade para conseguir o agendamento, além do tempo de espera para a consulta.

Olha, tem que marcar consulta [...] Aí demora alguns meses, mas quando a gente não está muito bem, eles fazem uma forma [...] pelo menos para mim eles atendem. (U2, ESF)

Então, você levanta às 5 horas da manhã para conseguir horário. Aí passam para tarde, aí você não é atendida, entendeu? Sempre para depois, nunca tem horário. (U4, UBST) 
TABELA 1: Distribuição da avaliação de aspectos que determinam a condição de acesso aos serviços de saúde por usuários da ESF e da UBST. Marília, 2012.

\begin{tabular}{|c|c|c|c|}
\hline Aspectos relacionados ao acesso & $\begin{array}{l}\text { Usuário UBS } \\
\text { (Media) }\end{array}$ & $\begin{array}{l}\text { Usuário ESF } \\
\text { (Media) }\end{array}$ & $P$ \\
\hline 1. Acha fácil conseguir uma consulta nessa unidade. & 4,62 & 4,16 & 0,091378 \\
\hline $\begin{array}{l}\text { 2. Esta unidade fica aberta após as } 18 \text { horas pelo menos um } \\
\text { dia/semana }\end{array}$ & 1,51 & 1,79 & 0,006537 \\
\hline $\begin{array}{l}\text { 3. Durante o período de funcionamento existe um telefone para } \\
\text { marcar consulta ou pedir informação. }\end{array}$ & 4,65 & 4,55 & 0,466583 \\
\hline $\begin{array}{l}\text { 4. Normalmente o(a) senhor (a) tem que esperar mais de } 30 \\
\text { minutos antes de ser atendido por um profissional de saúde. }\end{array}$ & 4,60 & 3,93 & 0,000953 \\
\hline $\begin{array}{l}\text { 5. Quando vem consultar nesta unidade o(a) senhor(a) tem que } \\
\text { perder o seu dia de trabalho. }\end{array}$ & 3,13 & 2,32 & 0,000043 \\
\hline $\begin{array}{l}\text { 6. Nas consultas mais recentes (no último mês) esta unidade } \\
\text { tinha todos os medicamentos que precisava. }\end{array}$ & 3,66 & 4,02 & 0,041289 \\
\hline $\begin{array}{l}\text { 7. Quando o(a) senhor(a) ou sua família precisa de algum } \\
\text { controle de saúde preventiva (vacinar, verificar pressão, exame } \\
\text { de rotina), vem a esta unidade. }\end{array}$ & 5,42 & 5,68 & 0,000331 \\
\hline $\begin{array}{l}\text { 8. Quando o(a) senhor(a) ou sua família tem algum problema } \\
\text { de saúde vem a esta unidade. }\end{array}$ & 5,34 & 5,56 & 0,006778 \\
\hline $\begin{array}{l}\text { 9. Exceto em caso de emergência o(a) senhor(a) ou sua família } \\
\text { tem que realizar uma consulta nesta unidade antes de consultar } \\
\text { com um especialista. }\end{array}$ & 5,22 & 5,55 & 0,000381 \\
\hline $\begin{array}{l}\text { 10. O (a) senhor (a) vai consultar com um especialista é } \\
\text { encaminhado por um profissional desta unidade. }\end{array}$ & 4,70 & 5,35 & 0,000279 \\
\hline $\begin{array}{l}\text { 11. Quando o (a) senhor (a) é encaminhado ao especialista os } \\
\text { profissionais desta unidade indicam ou discutem com o(a) } \\
\text { senhor (a)os possíveis lugares de atendimento. }\end{array}$ & 3,98 & 5,20 & 0,000000 \\
\hline $\begin{array}{l}\text { 12. Quando o(a) senhor (a) é encaminhado ao especialista os } \\
\text { profissionais desta unidade ajudam a marcar a consulta? }\end{array}$ & 4,53 & 5,39 & 0,000023 \\
\hline $\begin{array}{l}\text { 13. Quando o(a) senhor (a) é encaminhado ao especialista os } \\
\text { profissionais desta unidade fornecem informações escritas para } \\
\text { entregar ao serviço ou ao especialista. }\end{array}$ & 4,61 & 5,42 & 0,000026 \\
\hline $\begin{array}{l}\text { 14. O (a) senhor (a) retorna a este posto com as informações } \\
\text { escritas sobre o(s) resultado(s) da(s) consulta(s) com o(s) } \\
\text { especialista(s) }\end{array}$ & 3,98 & 5,08 & 0,000001 \\
\hline $\begin{array}{l}\text { 15. O(a) senhor (a) é informado nesta unidade sobre os } \\
\text { resultados da consulta com outro(s) especialista (a) }\end{array}$ & 4,20 & 5,19 & 0,000001 \\
\hline $\begin{array}{l}\text { 16. O(a) senhor (a)discute com o profissional desta unidade } \\
\text { sobre os resultados da consulta ao(s) especialista (a). }\end{array}$ & 3,53 & 4,77 & 0,000000 \\
\hline $\begin{array}{l}\text { 17. O (a) senhor (a) acha que os profissionais desta unidade } \\
\text { estão interessados na qualidade da sua consulta com o(a) } \\
\text { especialista? }\end{array}$ & 3,94 & 5,05 & 0,000000 \\
\hline
\end{tabular}

$\mathrm{Na}$ fala do usuário da ESF, é preciso considerar e destacar a demora de meses para conseguir uma consulta. É possível que, ao esperar tanto tempo após a necessidade sentida, alternativas tenham sido encontradas para a solução do problema. A demora no atendimento, além da baixa resolução, foi apontada em estudo realizado com usuários da ESF, na capital do Mato Grosso ${ }^{12}$.

Considera-se que a procura por assistência ocorre quando se acredita que alguma intervenção possa corrigir a situação de saúde julgada anormal, situação essa dependente de fatores como a oferta de assistência à saúde e o atendimento das expectativas do usuário diante dos serviços de saúde ${ }^{13}$.

Por outro lado, os profissionais, mesmo reconhecendo a queixa dos usuários, acreditam que por meio de outros mecanismos, como o acolhimento e a orientação, conseguem em parte dar encaminhamento dos casos, conforme descrito a seguir. 
TABELA 2: Distribuição da avaliação de aspectos que determinam a condição de acesso aos serviços de saúde por profissionais da ESF e da UBST. Marília, 2012.

\begin{tabular}{|c|c|c|c|}
\hline Acesso & $\begin{array}{l}\text { Prof. UBS } \\
\text { (Media) }\end{array}$ & $\begin{array}{l}\text { Prof. ESF } \\
\text { (Media) }\end{array}$ & $p$ \\
\hline $\begin{array}{l}\text { 1. Durante os últimos seis meses, com que frequência a unidade teve } \\
\text { adequada oferta de medicamentos essenciais? }\end{array}$ & 3,92 & 3,75 & 0,180551 \\
\hline $\begin{array}{l}\text { 2. Durante os últimos seis meses, com que frequência a unidade teve } \\
\text { adequado equipamento básico para cumprir com suas funções } \\
\text { essenciais. }\end{array}$ & 4,20 & 3,83 & 0,019033 \\
\hline $\begin{array}{l}\text { 3. Que porcentagem da população coberta pode obter uma consulta } \\
\text { médica (não urgente) no prazo de } 24 \text { horas? }\end{array}$ & 4,60 & 2,94 & 0,000000 \\
\hline $\begin{array}{l}\text { 4. A unidade fica aberta pelo menos um dia da semana depois das } 18 \\
\text { horas? }\end{array}$ & 2,37 & 1,67 & 0,016338 \\
\hline $\begin{array}{l}\text { 5. Durante o funcionamento da unidade existe um número de telefone } \\
\text { para marcar consultas ou pedir informações. }\end{array}$ & 5,36 & 5,18 & 0,335757 \\
\hline $\begin{array}{l}\text { 6. Quando a unidade está fechada, existe um número de telefone para } \\
\text { marcar consultas ou pedir informações? }\end{array}$ & 1,05 & 1,33 & 0,114684 \\
\hline $\begin{array}{l}\text { 7. Normalmente, o cliente/usuário tem que esperar mais de } 30 \\
\text { minutos na unidade antes de ser atendido por um profissional de } \\
\text { saúde? }\end{array}$ & 3,24 & 2,85 & 0,141862 \\
\hline $\begin{array}{l}\text { 8. Exceto em casos de emergência, é preciso uma consulta com } \\
\text { prestador do nível básico antes que um usuário busque outro nível de } \\
\text { atenção. }\end{array}$ & 5,14 & 5,25 & 0,079060 \\
\hline $\begin{array}{l}\text { 9. Existem normas definidas para transferências de informações sobre } \\
\text { pacientes entre os níveis de atenção. }\end{array}$ & 5,51 & 5,17 & 0,082771 \\
\hline $\begin{array}{l}\text { 10. Com que frequência os profissionais de saúde na sua unidade } \\
\text { utilizam normas definidas para referência e contra referência? }\end{array}$ & 5,51 & 5,24 & 0,019916 \\
\hline $\begin{array}{l}\text { 11. Quando um usuário/cliente precisar ser referido para outro serviço, } \\
\text { os profissionais de atenção básica discutem com ele ou indicam os } \\
\text { possíveis lugares de atendimento? }\end{array}$ & 4,97 & 5,46 & 0,000911 \\
\hline $\begin{array}{l}\text { 12. Existem mecanismos formais para marcar consultas com um } \\
\text { especialista? }\end{array}$ & 5,63 & 5,71 & 0,125339 \\
\hline $\begin{array}{l}\text { 13. Quando os pacientes são referidos para outro serviço, os } \\
\text { profissionais de atenção básica fornecem informações escritas? }\end{array}$ & 5,68 & 5,83 & 0,387164 \\
\hline $\begin{array}{l}\text { 14. Os profissionais de atenção básica recebem informações escritas } \\
\text { sobre os resultados das consultas referidas aos especialistas? }\end{array}$ & 4,52 & 3,81 & 0,000158 \\
\hline $\begin{array}{l}\text { 15. Existem normas definidas para a realização de exames } \\
\text { laboratoriais de complementação diagnóstica? }\end{array}$ & 5,58 & 5.41 & 0,158882 \\
\hline $\begin{array}{l}\text { 16. Existe supervisão periódica para revisar a necessidade de } \\
\text { referência aos outros níveis de atenção. }\end{array}$ & 4,36 & 3,97 & 0,134248 \\
\hline $\begin{array}{l}\text { 17. Os prontuários médicos sempre estão disponíveis quando o } \\
\text { profissional examina o usuário/paciente }\end{array}$ & 5,81 & 5,80 & 0,830998 \\
\hline
\end{tabular}

Bom, os usuários às vezes reclamam, mas nem sempre dá para agradar a todos, mas sempre são muito bem acolhidos e orientados. (P7, ESF)

Ressalta-se que, embora diferentes sujeitos e atores sociais compreendam, de formas variadas, o que são demandas por necessidades de saúde, essa compreensão precisa ser explicitada e colocada em diálogo ${ }^{14}$.

Estudo sobre a visão do usuário da ESF evidenciou que a localização geográfica, o vínculo e o acolhimento representam uma fortaleza, ainda que, por outro lado, prevaleça a visão reducionista, centrada na consulta médica e nas ações curativas ${ }^{15}$. Na rede assistencial de uma capital brasileira revelaram-se barreiras de acesso desde a atenção primária, porém de forma mais acentuada nos demais níveis ${ }^{16}$.

Reconhecidamente, os desafios a serem enfrentados perpassam as instâncias do financiamento, a força de trabalho e os modelos de gestão e de atenção. Há, ainda, necessidade de oferta de serviços de saúde em quantidade adequada, articulação entre os diferentes 
níveis de serviços com vistas à complementariedade, além de uma nova lógica de atenção à saúde, por meio de mudanças nas relações entre os trabalhadores e os usuários, principalmente pela escuta atentiva ${ }^{14}$.

Mesmo que a implantação da ESF tenha ampliado a oferta de serviços e aproximado os profissionais dos usuários, suas características fazem com que se aumente também a demanda. Em análise do que determina essa demanda, são apontados fatores variados, incluindo "necessidade sentida, fatores psicossociais, seguridade social, demografia, epidemiologia, utilização dos serviços, regulação e fatores culturais" $13: 127$. Os autores consideram que tais fatores se sobrepõem, resultado na demanda crescente e de difícil contenção.

\section{Porta de entrada sob distintas visões}

A unidade básica de saúde, como porta de entrada ao sistema, representa para os usuários uma condição que burocratiza o acesso, enquanto os profissionais entendem que a organização está adequada. A partir dessa divergência, é possível depreender que os profissionais são imbuídos de uma lógica objetiva de inclusão/exclusão do atendimento à demanda, o que nem sempre vai ao encontro dos desejos dos usuários que veem suas necessidades como únicas e prioritárias. No município em tela, para o atendimento da demanda, utiliza-se a classificação de risco, direcionada por critérios essencialmente objetivos que nem sempre coincidem com a necessidade sentida pelo usuário.

[...] meio difícil porque às vezes não tem médico. A gente tem que procurar o pronto socorro à noite, porque precisa de encaminhamento, e é uma burocracia. (U3, UBST)

A gente tem que se locomover para outras unidades, e a gente fica sem saber para aonde vai. Ou vai para Santa Casa, ou vai para o HC. Para aonde a gente vai, em casos de emergências, quando não tem médico? (U8, ESF)

É, a gente vem aqui para depois ser encaminhado. Se você não passa aqui, eles não atendem. Agora, lá, se você for na parte da tarde, assim perto das 5 horas, qualquer um que chegar consulta. (U1, ESF)

Pelas falas dos usuários, observa-se que ao se sentirem perdidos, por falta de funcionamento da porta de entrada, eles alteram os arranjos organizacionais e encontram escape para o atendimento de suas necessidades. Frente a isso, atropela-se o fluxo, levando à manutenção da sobrecarga de atendimento nos níveis de atenção de maior complexidade.

$\mathrm{Na}$ atual organização do sistema de saúde, a atenção primária constitui a porta de entrada prioritária e esse movimento de reorganização do modelo imprime racionalidade ao seu funcionamento. Parte-se da necessidade de maior adequação dos recursos destinados à saúde, considerando que muitos dos problemas podem ser resolvidos por meio de tecnologias leves e que os cenários de maior complexidade devem ser otimizados para atendimento de demandas específicas.

Em revisão teórica sobre o acesso aos serviços de saúde, também foram identificados avanços e retrocessos, confirmando que, mesmo que se tenha ampliado o acesso à rede básica, ainda permanecem dificuldades relacionadas ao acolhimento e à fragmentação da atenção à saúde ${ }^{17,18}$. Acrescenta-se que para garantir o acesso ao cuidado em saúde nos serviços públicos, deve-se contemplar tanto as relações interpessoais como as sócio-organizacionais ${ }^{19}$.

Pode-se considerar que os profissionais agem em conformidade com as normas e os fluxos impostos por essa racionalidade, sem levar em conta o que está implícito nas necessidades de cada usuário que procura por atendimento.

Em geral, tem alguma dificuldade, mas procuramos fazer com que ela tenha essa possibilidade. (P5,UBST)

Todos que procuram a unidade, de manhã, não saem sem atendimento. Só quando não tem recurso, as pessoas são encaminhadas para o hospital, mas todos são atendidos. (P4,ESF)

Reforça-se, dessa forma, a importância da participação ativa do usuário no processo de cuidado da atenção primária, com vistas à sua transformação $0^{20}$.

\section{Comprometimento dos fluxos de atendimento}

Nos depoimentos tanto dos usuários como dos profissionais, observa-se que o elenco de serviços está muito aquém das suas necessidades, especialmente no que se refere ao atendimento especializado. Há mais de uma década vem se ampliando a oferta de serviços de atenção primária por meio da implantação da ESF. Embora esse aspecto, sem dúvida, amplie a possibilidade de acesso, muitas vezes limita-se à porta de entrada, uma vez que os serviços de maior complexidade não receberam o mesmo tratamento. É possível que, sem a vazão dessa demanda, o usuário retorne frequentemente à porta de entrada e forme um ciclo de alta demanda e baixa resolutividade, o que causa desgaste aos profissionais no enfrentamento cotidiano para atender à população.

Acrescenta-se, ainda, a crença dos profissionais e da população no modelo centrado na doença e na complexa tecnologia, o que limita as possibilidades de avanço na perspectiva da promoção da saúde e do uso da tecnologia leve, pautada na escuta ampliada, no vínculo e na responsabilização como condição essencial para os avanços no atendimento às necessidades de saúde. As falas seguintes apontam para as dificuldades dos entrevistados quanto aos serviços de saúde aos quais legalmente têm direto, embora não sejam cientes disso.

Se eu ficasse contando com os medicamentos daqui, eu não me trataria nunca. Então eu compro os remédios e os exames eu faço particularmente. (U5, UBST) 
Encaminhamento... Eu esperei dois anos pra conseguir um neuro para minha filha, dois anos. (U7, UBST)

Eles fazem uma consulta para saber o que tenho [referindo-se ao serviço] [...] o resultado eu fui pegar depois de 15 dias. Deu tudo certo. (U2, UBST)

Associa-se às dificuldades encontradas pelos usuários em relação aos serviços, a baixa integração entre esses. Para ser atendido no serviço especializado, embora seja necessário um encaminhamento contendo a condição do usuário, a contrarreferência, via de regra, não é realizada pelo serviço especializado. É o próprio usuário que acaba trazendo a informação, o que pode dificultar a continuidade do cuidado. Estudo de revisão da literatura identificou tais aspectos entre as fragilidades relacionadas à atenção primária à saúde 21 .

As dificuldades na integração entre os serviços é mais observada pelos profissionais, talvez pelo fato de o usuário não visualizar a importância dela para a continuidade e a complementaridade no tratamento.

São poucos os serviços que fazem a contrarreferência. A gente fica sem saber se o paciente realmente foi, o que foi diagnosticado, a conduta, o seguimento [...] É ruim, principalmente quanto às especialidades. (P1, ESF)

Mesmo frente às dificuldades referentes aos fluxos de atendimento, ao se analisar a atenção primária na coordenação das redes de atenção, constatou-se que as fortalezas sobrepõem-se às fragilidades ${ }^{21}$. Quanto aos serviços de atenção secundária, para que possam contribuir com as práticas em rede, considera-se a necessidade de melhorar a capacidade dos serviços, de aprimorar as ferramentas de planejamento e de estabelecer protocolos ${ }^{22}$.

Vale ressaltar que a utilização das modalidades de pesquisa qualitativa e quantitativa, para analisar um fenômeno, representa uma atividade complexa e, algumas vezes, contraditória. No entanto, buscou-se lidar com tal condição na perspectiva da complementaridade.

\section{ConClusão}

$\mathrm{Na}$ verificação da acessibilidade, constata-se que a maioria dos aspectos pontuados tende a uma avaliação positiva, sendo que os usuários da ESF demonstram maior satisfação quando comparados com os usuários da UBST. Referindo-se aos profissionais, observa-se que os da UBST os avaliam como mais positivos.

Quanto à localização da unidade de saúde, tanto profissionais como usuários consideram-na adequada para o atendimento das necessidades. No entanto, evidencia-se a demora em marcar consulta médica, fato que se revela mais intenso na ESF quando com- parada com a UBST. Agrega-se a tal aspecto a restrição nos horários de funcionamento das unidades.

Referindo-se à atenção primária como porta de entrada, os usuários a consideram, em alguns momentos, como um empecilho ao atendimento desejado. Os profissionais compreendem que o atendimento está adequado às necessidades, possivelmente por operarem pautados na objetividade do sistema de classificação de risco, enquanto os usuários procuram os serviços de saúde pelo desejo de um atendimento médico imediato.

O elenco de serviços é considerado insuficiente para a totalidade dos participantes do estudo, o que é observado principalmente pela demora do atendimento, anos, e, muitas vezes pela impossibilidade de obter atendimento especializado e exames específicos. Quanto à integração entre os serviços de saúde, nos diferentes níveis de atenção, constata-se que, embora os usuários sempre cheguem aos serviços de maior complexidade por meio de encaminhamento, a contrarreferência praticamente inexiste. Esse aspecto é reconhecido principalmente pelos profissionais, uma vez que os usuários parecem não ter clara essa necessidade.

Conclui-se que tanto a UBST como a ESF apresentam fortalezas e fragilidades que demandam novos modos de organização e gestão, considerando, essencialmente, os aspectos subjetivos envoltos nas necessidades de cada usuário.

Pontua-se como fragilidade do estudo o fato de ter sido realizado em um único município, o que impede generalizações, considerando que cada município adota diferentes formas para organizar a rede de atenção. Espera-se, no entanto, que os resultados proporcionem acréscimos ao conhecimento sobre a organização e ao funcionamento dos serviços de saúde, reforçando de suas potencialidades e para superar os desafios.

\section{REFERÊNCIAS}

1.Governo Federal (BR). Lei 8.080, de 19 de setembro de 1990. Dispõe sobre as condições para a promoção, proteção e recuperação da saúde, a organização e o funcionamento dos serviços correspondentes e dá outras providências. Brasília (DF); Poder Executivo; 1990.

2.O'Donnell O. Access to health care in developing countries: breaking down demand side barriers. Cad Saúde Pública. 2007; 23: 2820-34.

3.Starfield B. Atenção primária: equilíbrio entre necessidades de saúde, serviços e tecnologia. Brasília (DF): Ministério da Saúde; 2002.

4.Giovanella L, Fleury S. Universalidade da atenção à saúde: acesso como categoria de análise. In: Eibenschutz C, organizador. Política de saúde: o público e o privado. Rio de Janeiro: Fiocruz; 1996. p. 177-98. 
5.Jesus WLA, Assis MMA. Revisão sistemática sobre o conceito de acesso nos serviços de saúde: contribuições do planejamento. Cienc Saúde Coletiva. 2010; 15: 161-70. 6.Pessoto UC, Heimann LS, Boaretto RC, Castro IEN, Kayano J, Ibanhes LC, et al. Desigualdades no acesso e utilização dos serviços de saúde na região metropolitana de São Paulo. Cienc Saúde Coletiva. 2007; 12: 351-62. 7.Travassos C, Viacava F. Acesso e uso de serviços de saúde em idosos residentes em áreas rurais, Brasil, 1998 e 2003. Cad Saúde Pública. 2007; 23: 2490-502.

8.Cunha ABO, Vieira-da-Silva LM. Acessibilidade aos serviços de saúde em um município do Estado da Bahia, Brasil, em gestão plena do sistema. Cad Saúde Pública. 2010; 26: 725-37.

9.Minayo MCS. O desafio do conhecimento: pesquisa qualitativa em saúde. 12a ed. São Paulo: Hucitec; 2010. 10.Barbetta PA. Estatística aplicada às ciências sociais. 3a ed. Florianópolis (SC): UFSC; 1999.

11.Oliveira EFT, Grácio MCC. Análise a respeito do tamanho de amostras aleatórias simples: uma aplicação na área de ciência da informação. DataGramaZero [Internet] 2005 [citado em 20 jan 2014]. 6(3). Disponível em: http://www.dgz.org.br/ago05/Art_01.htm

12.Corrêa ACP, Ferreira F, Cruz GSP, Pedrosa ICF. Acesso a serviços de saúde: olhar de usuários de uma unidade de saúde da família. Rev Gaúcha Enferm. 2011; 32: 451-7. 13.Zucchi P, Del Nero C, Malik AM. Gastos em saúde: os fatores que agem na demanda e na oferta dos serviços de saúde. Saude Soc. 2000; 9: 127-50.

14.Ministério da Saúde $(\mathrm{Br})$. Acolhimento à demanda espontânea. Brasília (DF): Ministério da Saúde; 2011.

15.Nery AA, Carvalho CGR, Santos FPA, Rodrigues VP. Saúde da família: visão dos usuários. Rev enferm UERJ. 2011; 19: 397-402.

16.Souza FOS, Medeiros KR, Gurgel Junior GD, Albuquerque $\mathrm{PC}$. Do normativo à realidade do Sistema
Único de Saúde: revelando barreiras de acesso na rede de cuidados assistenciais. Cienc Saúde Coletiva [ScieloScientific Electronic Library Online] 2014 [citado em 20 set 2014]. 19:1283-93. Disponível em: http://www. scielo.br/pdf/csc/v19n4/1413-8123-csc-19-04-01283.pdf 17.Assis MMA, Jesus WLA. Acesso aos serviços de saúde: abordagens, conceitos, políticas e modelo de análise. Cienc Saúde Coletiva. 2012; 17: 2865-75.

18.Macedo CA, Teixeira ER, Daher DV. Possibilidades e limites do acolhimento na percepção de usuários. Rev enferm UERJ [Internet] 2011 [citado em 20 set 2014]. 19: 457-62. Disponível em: http://www.facenf.uerj.br/ v19n3/v19n3a20.pdf

19.Barbosa SP, Elizeu TS, Penna CMM. Ótica dos profissionais de saúde sobre o acesso à atenção primária à saúde. Cienc Saúde Coletiva [ScieloScientific Electronic Library Online] 2013 [citado em 20 set 2014]. 18:2347-57. Disponível em: http:// www.scielosp.org/scielo.php?script =sci_arttext\&pid $=$ S1413-81232013001600019

20.Clares JWB, Silva LMS, Dourado HHM, Lima LL. Regulaçao do acesso ao cuidado na atenção primária: percepção dos usuários. Rev enferm UERJ. 2011; 19: 604-9. 21.Rodrigues LBB, Silva PCS, Peruhype RC, Palha PF, Popolin MP, Crispim JA, et al. A atenção primária à saúde na coordenação das redes de atenção: uma revisão integrativa. Cienc Saúde Coletiva [Scielo-Scientific Electronic Library Online] 2014 [citado em 20 set 2014]. 19:343-52. Disponível em: http://www.scielo.br/pdf/csc/ v19n2/1413-8123-csc-19-02-00343.pdf

22.Erdmann AL, Andrade SR, Mello ALSF, Drago LC. $A$ atenção secundária em saúde: melhores práticas na rede de serviços. Rev Latino-Am Enfermagem [ScieloScientific Electronic Library Online] 2013 [citado em 20 set 2014]. 21 (esp):131-9. Disponível em: http://www. scielo.br/pdf/rlae/v21nspe/pt_17.pdf 